\title{
Ruthenium Organometallic-bromophenol Blue Pairs in Hydrogel Sensing Matrix for Dissolved Ammonia Förster Resonance Energy Transfer Sensor
}

\author{
Zainiharyati Mohd Zain, ${ }^{1,2 *}$ Aiman Sajidah Abd Aziz, ${ }^{1}$ Mohd Norzaliman Mohd Zain, ${ }^{1}$ \\ Muhammad Syargawi Abdullah, ${ }^{1}$ and Siti Zulaikha Rejal ${ }^{1}$ \\ ${ }^{1}$ Photonics Department, MIMOS Berhad, Technology Park Malaysia, 57000 Kuala Lumpur, Malaysia \\ ${ }^{2}$ Faculty of Applied Sciences, Universiti Teknologi MARA, 40450, Shah Alam, Selangor, Malaysia
}

(Received December 19, 2017 ; accepted April 11, 2018)

Keywords: dissolved ammonia sensor, hydrogel sensing membrane, optical FRET

Monitoring dissolved ammonia (DA) in tilapia fish farming ponds is important because a DA concentration as low as 100 parts per billion (ppb) depresses tilapia food intake and growth. Hence, a DA sensor capable of sub-ppm-level detection based on the Förster resonance energy transfer (FRET) of a single donor-acceptor pair has been developed to determine DA in tilapia breeding ponds. Certain mole ratio amounts of ruthenium organometallic complex (donor) to bromophenol blue (acceptor) were immobilized in hydrogel, polyvinyl chloride, and polysiloxanes membrane matrices. The hydrogel membrane matrix showed the best performance. A DA sensitivity at the ppb level has been achieved on a membrane of 2-3 $\mu \mathrm{m}$ thickness. The FRET sensing membrane performed reversibly with a response time of $10 \mathrm{~min}$ at $100 \mathrm{ppb}$ DA in phosphate buffer ( $\mathrm{pH}$ 11) with $100 \%$ (8 ppm) dissolved oxygen content in the solution. The $\mathrm{pH}$ and other amines did not interfere with the selectivity toward DA. Hence, the membrane has been applied to determine the DA concentration in tilapia fish ponds with $80 \%$ accuracy as compared with the Nessler method.

\section{Introduction}

Dissolved ammonia (DA) is naturally found and is an important species present in landed fish and marine aquaculture ponds. High concentrations of DA in fish farming ponds originate from environmental pollution due to agricultural and industrial wastes and solid waste leachates, resulting in unhealthy fish and marine aquatic species and affecting the quality and production of marine products. ${ }^{(1)}$ Continuous monitoring of DA, $\mathrm{pH}$, dissolved oxygen (DO), temperature, and salinity is important because of their possible effects on organisms' health, feed utilization, growth rates, and stocking densities. For example, an increase in ammonia level results in potentially fatal pathophysiological damage to the gills and kidneys, and neurotoxicity, hyperventilation, and convulsions have been observed. ${ }^{(2)}$ The indophenol blue or Berthelot reaction is commonly used for water analysis. To date, it has been developed into

*Corresponding author: e-mail: zainihar@salam.uitm.edu.my http://dx.doi.org/10.18494/SAM.2018.1841 
an integrated microfluidic system but requires significant consumptions of reagents and works slowly. ${ }^{(3)}$ Electrochemistry is a popular analytical approach but experiences interference from the salinity of water samples and marine corrosion. Many methods based on optical intensity (fluorescence and absorption) have been developed for ammonia detection, ${ }^{(4-8)}$ but they may suffer from optical path displacement and photobleaching of the optical probe. A phasebased method has been introduced to overcome these drawbacks, in which the optimized light intensity thus reduces sensor photobleaching. Moreover, the Förster resonance energy transfer (FRET) method is more robust with stable measurement reading than the intensity-based method as it is less sensitive to the optical alignment of reflected light from the optical probe onto the spectrophotometer.

The principle behind the optical chemical ammonia sensor described here is based on the Förster distance of the luminophore or donor (ruthenium organometallic complexes) from the chromophore or acceptor (e.g., pH-sensitive indicator). An optimum Förster distance results in the efficient overlap of excitation and emission spectra of both donor and acceptor because the luminophore possesses a longer decay time, and nonradiative energy is transferred to the acceptor after it reacts with DA. The frequency domain method of the measuring phase and modulation is advantageous because the luminophore photobleaching usually occurs in an intensity-based measurement. The concept of dynamic quenching allows the determination of DA concentration based on the observed phase shift of the decay time of the total emission signal. This mechanism is termed dynamic or collisional quenching, where the quenching agent (Q) (acceptor) accepts nonradiative energy transfer from the donor. ${ }^{(9)}$ In this study, an optochemical sensing probe was embedded on a bundle of fiber optic cables connected to a time/frequency detector, which minimize displacement of the optical path length. The advantages of a FRETbased optical chemical ammonia sensor are that it is relatively cheap to implement, enables rapid detection, and is robust in marine environments compared with an optical intensity-based setup.

\section{Materials and Methods}

\subsection{Materials}

Ammonia solutions, polyvinyl chloride (PVC), bromophenol blue sodium salt (BPB), hydrogel D7, dioctyl sebacate (DOS), hydrochloric acid, $N, N$-dimethylformamide (DMF), ethanol (EtOH), methanol (MeOH), 2-nitrophenyl octyl ether (NPOE), tetrahydrofuran (THF), tetraethyl orthosilicate, and tetramethyl orthosilicate (TEOS) were purchased from SigmaAldrich, Germany. The ruthenium complex $\left[\mathrm{Ru}(\mathrm{dpp})_{3}\right]$ was obtained from American Elements, USA. All chemicals were of analytical grade. DA test solutions were freshly prepared and verified using indophenol reagent (Photometer 7500) before use.

\subsection{Optical membrane preparation}

$0.4 \mathrm{mg}$ of $\mathrm{BPB}, 0.3 \mathrm{mg}$ of ruthenium, and $0.4 \mathrm{mg}$ of hydrogel D7 were weighed inside a vial. Then, $4 \mathrm{~mL}$ of DMF was pipetted and transferred into the vial. The vial containing 
the cocktail was sealed with parafilm and aluminium foil. Then, the cocktail was sonicated for $2 \mathrm{~h}$. An $\mathrm{O}$ ring of $0.6 \mathrm{~cm}$ diameter was arranged on the biaxially-oriented polyethylene terephthalate (BoPET) as shown in Fig. 1(c). $10 \mu \mathrm{L}$ of the cocktail solution was pipetted using a micropipette and was drop-cast onto the BOPET to ensure that the cocktail was on the center of the $\mathrm{O}$ ring. A transparent membrane 2 to $4 \mu \mathrm{m}$ thick with an internal diameter of $6 \mathrm{~mm}$ was produced as shown in Fig. 1(a). The sensing membrane was mounted and tightly sealed with polytetrafluorethylene (PTFE) and positioned at the end of an optical fiber tip as shown in Fig. $1(b)$.

\subsection{Instrumentation}

The fluorescence emissions were measured using a high-resolution spectrometer (HR4000 UV-VIS, Ocean Optics, USA). The excitation light source was emitted by a blue pig-tailed LED (LE-3B, WT\&T, South Korea) at $455 \mathrm{~nm}$ triggered from a custom controller board. The controller allowed the blue LED to be triggered externally by a function generator (AFG3102, Tektronix, USA) at $45 \mathrm{kHz}$. This initial modulation phase reference signal was measured using a lock-in amplifier (Signal Recovery, 7265, Ametek Scientific Instrument, USA). The sensor membrane was attached at the end of a bifurcated fiber probe (BIFBORO-1000-2, Ocean Optics, USA). The blue light source propagated through this bifurcated fiber and excited the sensor membrane. The fluorescence emission was measured concurrently by a photodetector and converted to an electrical signal. The returning signal was detected by the lock-in amplifier. A set of optical bandpass filters (FD1B, FD1R; Newport, USA) was placed before the photodetector to ensure that only red fluorescence emissions coming from the sensor membrane were measured and to increase the signal-to-noise ratio (SNR). The experimental setup is depicted in Fig. 2. The spectra were observed using a UV spectrophotometer (Lambda 35, Perkin Elmer, USA) and spectrofluorometer (LS 55 Perkin Elmer, USA). GraphPAd Prism version 6 was used to manage the data.

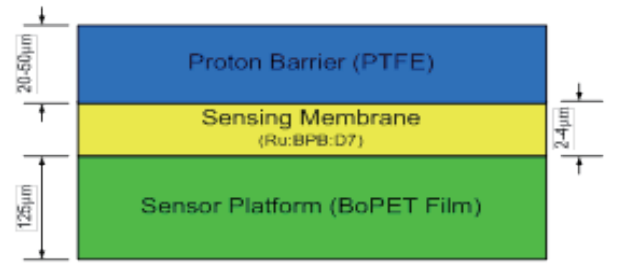

(a)

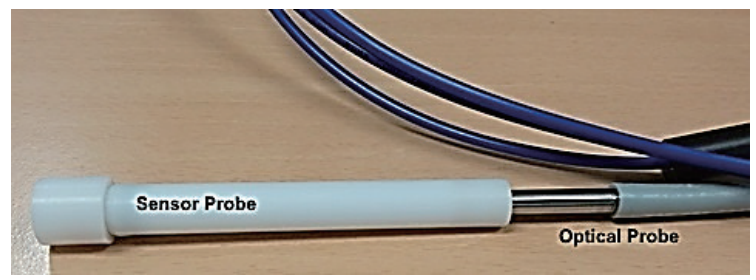

(b)

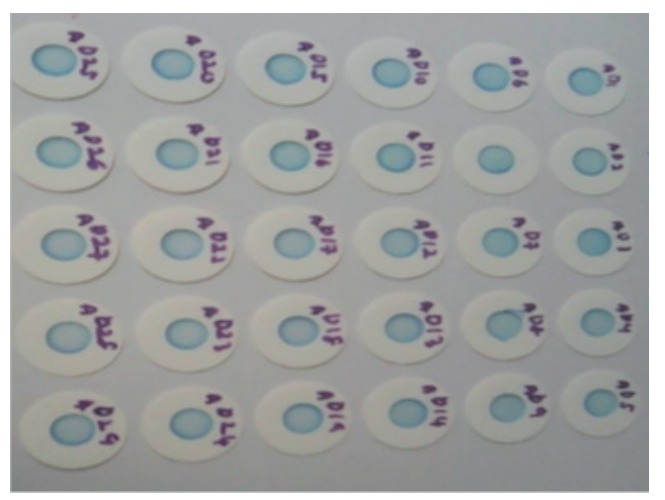

(c)

Fig. 1. (Color online) (a) Schematic diagram of DA sensing membrane, (b) sensor probe, and (c) an O-ring entrapped the sensing cocktail that was drop-cast on the BOPET producing a 2- $\mu$ m-thick transparent optical membrane. 


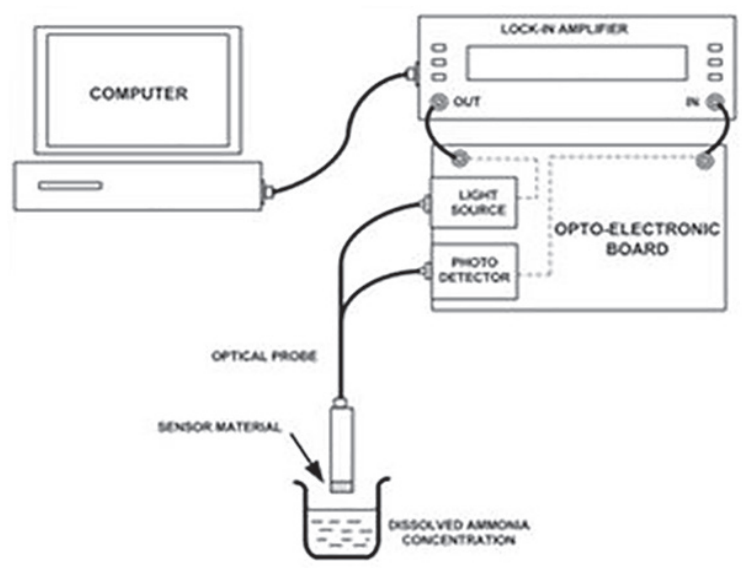

Fig. 2. Experimental setup.

\section{Results and Discussion}

\subsection{Donor emission and acceptor absorption spectra}

DA diffused into the sensing membrane, reacted with the protonated $\mathrm{pH}$ indicator (IndH), and deprotonated the indicator (Ind) to form the ammonium ion $\left(\mathrm{NH}_{4}{ }^{+}\right)$as a counter ion, as in Eq. (1).

$$
\mathrm{NH}_{3}+\mathrm{IndH} \rightarrow \mathrm{NH}_{4}^{+}+\mathrm{Ind}^{-}
$$

The unprotonated indicator has an absorbance maximum at $609 \mathrm{~nm}$ [Fig. 3(a)], while the protonated form was observed at $440 \mathrm{~nm}$ in agreement with Meier et al. ${ }^{(9)}$ The effect of DA concentration on the UV absorbance of both protonated and unprotonated indicators is shown in Fig. 3(b). As the DA concentration increases, the absorbance of the deprotonoted indicator increases and that of the protonated indicator decreases. A DA absorbance calibration curve showed a linear relationship in agreement with Beer's Law. Interestingly, excitation of Ru $(\mathrm{dpp})_{3}$ in the range from 450 to $470 \mathrm{~nm}$ was observed using UV-vis. This excitation wavelength range was further chosen as the excitation wavelength in the spectrofluorometer to obtain approximately $\mathrm{Ru}(\mathrm{dpp})_{3}$ maximum emission intensity at $609 \mathrm{~nm}$. An efficient overlap of the $\mathrm{Ru}(\mathrm{dpp})_{3}$ emission and unprotonoted indicator absorption enables an efficient energy transfer in the FRET system. The overlapped emission and absorption spectra increased as the DA concentration increased. Spectral overlap was verified using spectrophotometers before using the FRET system because FRET failure alone would not enable the evaluation of incorrect donor-acceptor orientation or donor-acceptor distance.

\subsection{Optimization of DA FRET optical chemical sensor}

Several parameters of transmissive optical membrane properties were identified to obtain good FRET. They are the polymer chosen as the donor-acceptor immobilization medium, 


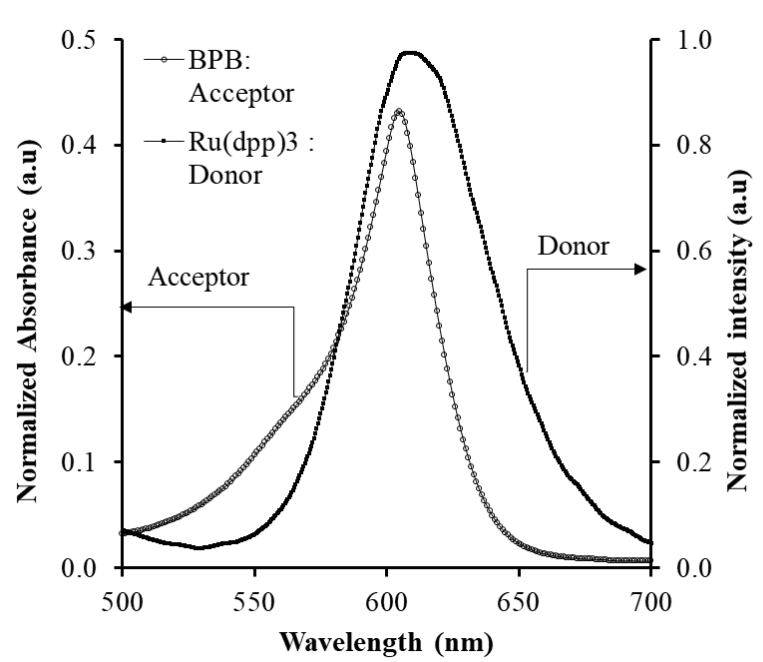

(a)

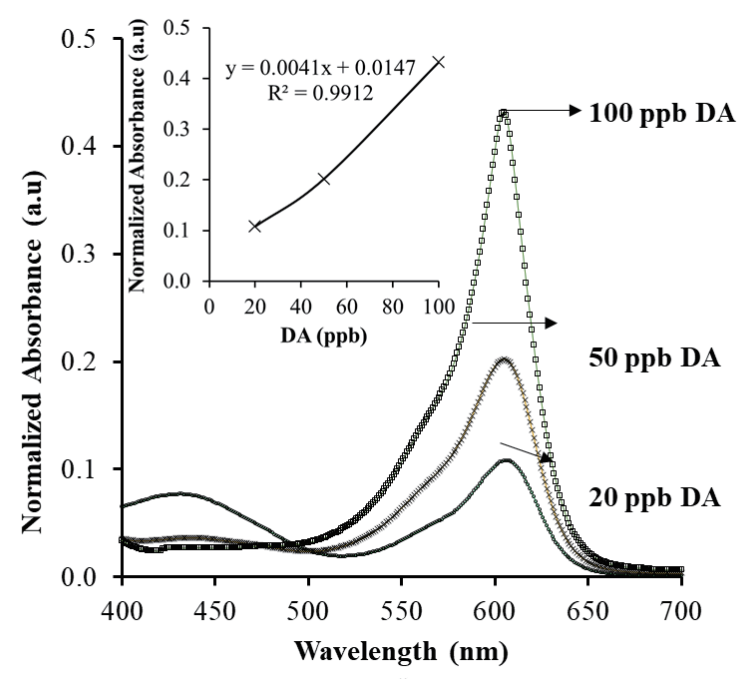

(b)

Fig. 3. (Color online) (a) Spectral integral or spectrum overlap between the fluorescence emission of the ruthenium complex and the absorbance of the deprotonated bromophenol blue and (b) effect of DA absorbance.

solvents for polymer membranes, donor-acceptor ratiometric loading, and membrane thickness. The type of polymeric membrane as the donor-acceptor immobilization medium was selected by comparing the quenching constant $\left(K_{D}\right)$ values of the polymers studied. The $K_{D}$ value is derived from the Stern-Volmer equation [Eq. (2)] below. In this equation, $\tau_{o}$ and $\tau$ are the lifetimes in the absence and presence of the quencher, respectively, $k_{q}$ is the quenching constant, $Q$ is the concentration of the quencher, and $K_{D}$ is the Stern-Volmer dynamic quenching constant.

$$
\frac{\tau_{o}}{\tau}=1+k_{q} \tau_{0}[Q]=1+K_{D}[Q]
$$

As tabulated in Table 1, the polysiloxane sol-gel matrix exhibited the lowest quenching value. The PVC polymer showed a moderate value and the polyether polyurethane (hydrogel) has the highest Stern-Volmer dynamic quenching constant, which makes it advantageous as a membrane matrix for luminescent probes with longer decay times and results in high sensitivity towards DA. The high water content of hydrogels renders them biocompatible and allows DA diffusion through the polymer network. ${ }^{(11)}$ The hydrogels were dissolved in tetrahydrofuran in which the $\mathrm{Ru}(\mathrm{dpp})_{3}$ emission peak was maintained at $\lambda_{\max }=609 \mathrm{~nm}$. Other solvents, namely, EtOH, MeOH, and DMF, shifted the $\lambda_{\max }$ of the Ru (dpp) 3 emission peak to 615, 618, and 627 $\mathrm{nm}$, respectively, which resulted in an insufficient overlap of donor emission and acceptor absorption spectra, thereby decreasing the FRET efficiency as shown in Fig. 4.

A PVC membrane plasticized by DOS performed better than NPOE as NPOE contains nitro groups that quench $\mathrm{Ru}(\mathrm{dpp})_{3}$. However, the inhomogeneity of water-saturated plasticized PVC caused not only the red emission light propogated into the fiber optical core (FOC) but also decreased FRET efficiency between donor and acceptor. Sol-gels have become increasingly 
Table 1

Stern-Volmer quenching constants of matrices studied.

\begin{tabular}{lc}
\hline Polymer matrix & Quenching constant $\left(K_{D}\right)$ \\
\hline Polysiloxanes sol-gel & 0.0968 \\
PVD & 0.2720 \\
Hydrogel & 0.4927 \\
\hline
\end{tabular}

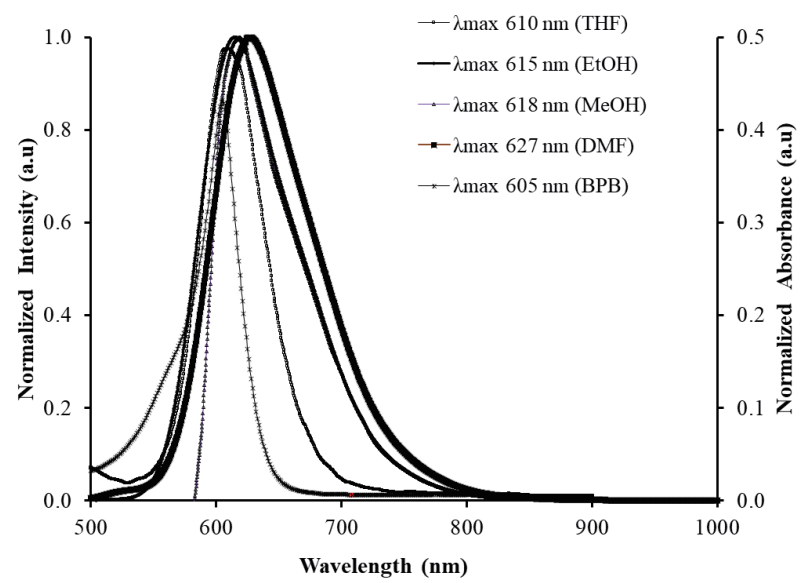

Fig. 4. (Color online) Spectral integral of ruthenium dissolved in various solvents and deprotonated bromophenol blue.

promising membrane matrices for optical sensors. Therefore, we synthesized a sol-gel-based membrane matrix as described elsewhere with minor modifications. ${ }^{(12)}$ The FRET signal was not continuous and displayed a high SNR owing to the rigidity of the sol-gel that resulted in poor transmission. The sol-gel rigidity can be resolved by manipulating the hydrolysis and condensation of tetraethoxisilane in the sol-gel network. ${ }^{(13)}$

\subsection{Analytical performance of DA sensor}

The phase shift and modulation of emissions depend on the relative values of the lifetime and the light source modulation frequency. A modulation frequency of $45 \mathrm{KHz}$ was applied and a $\mathrm{Ru}(\mathrm{dpp})_{3}$ luminescent decay time of 3 to $4 \mu \mathrm{s}$ in the absence of DA was obtained. A series of DA concentrations were spiked into test solutions to establish a linear relationship $\left(y=1.5144 x-1.4691, R^{2}=0.9973\right)$ in the DA concentration range of $0-100 \mathrm{ppb}$ with shifted phase angle $\left(4^{\circ}\right)$. It was observed that increased the DA concentration resulted in an increased shifted phase angle because the $\mathrm{Ru}(\mathrm{dpp})_{3}$ donor lifetime was quenched by the BPB acceptor. An overall phase-shifted signal change of $3.7 \pm 1.0^{\circ}$ was observed over the DA concentration range of 0 to $100 \mathrm{ppb}$. The hydrogel host matrix itself was not responsive to either $\mathrm{pH}$ or DO. The $\mathrm{Ru}(\mathrm{dpp})_{3}$ donor and $\mathrm{BPB}$ acceptor used in the sensor matrix are prone to $\mathrm{pH}$ and oxygen interferences. The sensor was exposed to a different level of $\mathrm{pH}$ buffer ranging from $\mathrm{pH} 4$ to 11. The sensor exhibited a nonlinear phase-shifted signal, and lifetime responses indicated that 
the sensor has low sensitivity to $\mathrm{pH}$. Additionally, a phosphate buffer of $\mathrm{pH} 11$ gave optimum performance owing to the nature of the dissociation of the ammonium ion in aqueous solution at $\mathrm{pHs}$ above $\mathrm{pH}$ 9.7. The sensing membrane did not respond to $\mathrm{pH}$ changes from $\mathrm{pH} 4$ to 9 as the sensing membrane was sealed with a $50-\mu \mathrm{m}$-thick PTFE membrane, which overcame cation interferences. This sensor is fully reversible as shown by the forward and backward slopes that were statistically compared using ANOVA $(P<0.05)$.

Generally, some types of ammonia sensors based on $\mathrm{pH}$ indicators also respond to other uncharged amines. Hence, we examined the interference effects of dicyclohexylamine ( $p K_{a}$ : $10.40)$, urea $\left(p K_{a}: 0.10\right)$, and butylamine $\left(p K_{a}: 10.60\right)$ towards DA $\left(p K_{a}: 9.25\right)$ at the same concentration, which is $500 \mathrm{ppb}$. All these species did not interfere with the DA sensor, and the $\log K_{\text {opt }}$ values determined (defined as the log of the ratio of the interfering signal and analyte signal at the same concentration) were 1.17 for ammonia-dicyclohexylamine, 1.18 for ammoniaurea, and 1.2 for ammonia-butylamine. The optical DA sensor has been demonstrated to be operational in the specified range of 10 to $40{ }^{\circ} \mathrm{C}$. The sensitivity slope of the DA sensor became lower at temperatures above $40^{\circ} \mathrm{C}$. The drawback of using $\mathrm{Ru}(\mathrm{dpp})_{3}$ as a donor is that $\mathrm{Ru}(\mathrm{dpp})_{3}$ is prone to oxygen quenching. A $t$-test ANOVA was used to compare the effect of sensors for a series of DA concentrations at two oxygen saturation levels, i.e., 0 and $100 \%$ DO. An $\mathrm{H}_{\mathrm{o}}$ hypothesis was defined as no sensor sensitivity slope differences observed in 0 and $100 \% \mathrm{DO}$, while an $\mathrm{H}_{\mathrm{A}}$ hypothesis stated the opposite. It was observed that the $\mathrm{H}_{\mathrm{o}}$ hypothesis was true and sensors showed no significant effects in the presence of DO $(P$-value $>0.05)$. The donor lifetime was proportionally decreased under both DO conditions. The stability of the DA sensor was measured by leaving multiple DA sensors in a $50 \mathrm{ppb}$ DA solution for $9 \mathrm{~d}$. The DA sensor donor lifetime decreased from 3.81 to $3.25 \mu$ s after being exposed for $4 \mathrm{~d}$ and then remained stable at $3.11 \mu$ s until day 9. This could be due to the photobleaching of the BPB dye, which was demonstrated in the absorbance study.

\subsection{Sensor deployment}

Ammonia is toxic to blue tilapia at concentrations above $2.5 \mathrm{ppm},{ }^{(14)}$ at which level it causes tissue damage, which later causes susceptibility to diseases. The DA sensor developed may be used to determine the actual DA content in a controlled tilapia fish breeding pond and was verified using the Nessler standard method to comply with test B in ASTM146-05. ${ }^{(14)}$ The ammonia sensors have shown $80 \%$ accuracy, which is comparable to that of a standard Nessler method. Nessler's reagent was used to determine the concentration of ammonia in the sample solutions using the direct nesslerization technique. The test solutions were added with $1 \mathrm{ml}$ of Nessler reagent and analyzed using a UV-vis spectrophotometer.

\section{Conclusions}

A DA sensor based on FRET lifetime and capable of determining sub-ppm concentrations has been successfully developed for in situ water monitoring in tilapia fish breeding ponds. Although the Nessler method was used as a standard analytical method for DA, this sensor has 
demonstrated better analytical performance and a low detection limit (10 ppb) with a sensor accuracy of $80 \%$, which is comparable to that of a standard Nessler procedure. The hydrogel outperformed PVC and sol-gel as a polymeric membrane matrix where immobilized $\mathrm{Ru}(\mathrm{dpp})_{3}$ and bromophenol blue pairs worked well as the donor and acceptor, respectively, in the FRET system. In addition, the solid-state sensing material optimization study showed sufficient FRET donor-acceptor overlapping spectra with a high quenching constant; $K_{D}$ of 0.4927 was achieved with a hydrogel $\mathrm{Ru}(\mathrm{dpp})_{3}$-BPB-based sensing matrix. The FRET optical sensing system was optimized by studying frequency modulation, sensitivity, and interference with respect to $\mathrm{pH}$, oxygen, and amines. The DA sensor has been used to measure the DA content of a controlled tilapia fish breeding pond with a measured DA level below $500 \mathrm{ppb}$.

\section{Acknowledgments}

Z.M. Zain is grateful to the Staff Industrial Attachment Scheme granted from the University Technology MARA Shah Alam Malaysia.

\section{References}

1 D. J. Randall and T. K. N. Tsui: Mar. Pollut. Bull. 45 (2002) 17.

2 D. J. Randall and Y. K. Ip: Resp. Physiol. Neurobiol. 154 (2006) 216.

3 L. O. C. Šraj, M. I. G. S. Almeida, S. E. Swearer, S. D. Kolev, and I. D. McKelvie: TrAC Trends Anal. Chem. 59 (2014) 83.

4 T. Abies, B. Ungerböck, I. Klimant, and T. Mayr: Chem. Cent. J. 6 (2012) 124.

5 K. Waich, T. Mayr, and I. Klimant: Talanta 77 (2008) 66.

6 J. L. Wojkiewicz, V. N. Bliznyunk, S. Carquiny, N. Elkamchi, N. Redon, T. LAsri, A. A. Pud, and S. Reynaud, Sens. Actuators, B 160 (2011) 1394.

7 A. Yimit, K. Itoh, and M. Murabayashi: Sens. Actuators, B 88 (2003) 239.

8 Y. B. Cho, S. H. Jeong, H. Chun, and Y. S. Kim: Sens. Actuators, B 256 (2018) 167

9 J. R. Lakowicz: Principles of Fluorescence Spectroscopy (Springer, New York, 2006) 3rd ed.

10 B. Meier, T. Werner, I. Klimant, and O. S. Wolfbeis: Sens. Actuators, B 29 (1995) 240.

11 D. Buenger, F. Topuz, and J. Groll: Prog. Polym. Sci. 37 (2012) 1678.

12 C. Preininger and G. J. Mohr: Anal. Chim. Acta. 342 (1997) 207.

13 X. Yang, L. Zhu, Y. Chen, B. Bao, J. Wu, and W. Zhou: Appl. Surf. Sci. 376 (2016) 1.

14 A. Ç. K. Benli, G. Köksal, and A. Özkul: Chemosphere 72 (2008) 1355.

15 B. C. Gordall: Treat. Water Sci. 3 (2011) 263.

\section{About the Authors}

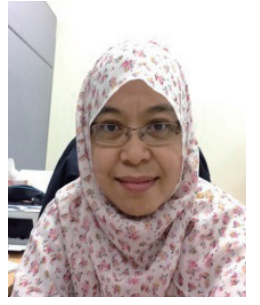

Zainiharyati Mohd Zain obtained a Bachelor of Industrial Chemistry degree with a minor in Management in 1997, a MSc (Chemistry) degree in 2005, and a Ph.D. (Electroanalytical Chemistry) degree in 2010 from Universiti Sains Malaysia. Her research interests are mainly on sensors ranging from implantable microelectrodes for neurochemicals, DNA biosensors for cancer genes, optical FRET-based sensors, electrochemical sensors for gunshot residues, and immunosensors for Alzheimer's biomarkers. 


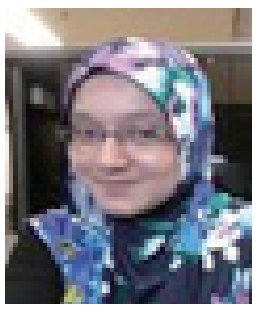

Aiman Sajidah Abd Aziz received a bachelor degree in Biochemical Engineering from Universiti Kebangsaan Malaysia, Malaysia. From 2006 to 2007, she was a research assistant at the Micro-electromechanical System (MEMS) Division, MIMOS Berhad. In 2007, she joined the Electrochemical Sensor and Microenergy Department, continued her research work in polymeric sensing materials, sensor transducers for ion-selective electrodes (ISE), and self-powered remote sensors through electrochemical and solar cell energy harvesters. Later in 2013, she initiated research focusing on optical sensing materials. Her research interests include optical and chemical sensing materials and sensor applications. She holds 15 patents. She was a recipient of the Design for Six Sigma (DFSS) Green Belt from Motorola University.

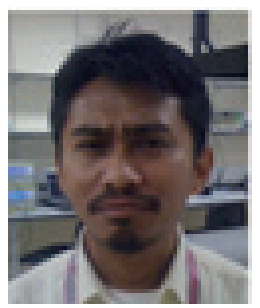

Mohd Norzaliman Mohd Zain received a B.Sc. degree in Physics and M.Sc. degree in micro-electronics and nano-engineering from Universiti Kebangsaan Malaysia. He joined MIMOS Berhad in 1998 as a senior researcher. His research interests include photonics sensors, quantum key distribution (QKD), and free space optics.

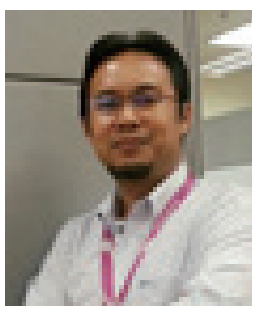

Muhanmmad Syargawi Abdullah received a B.Eng. degree in ElectricalElectronics Engineering from Universiti Teknologi Malaysia in 2001 and a M.Eng. degree in Electrical Engineering from Universiti Tenaga Nasional, Kajang, Selangor, in 2011. From 2001 to 2004, he was an R\&D engineer with the IBS Technology Sdn. Bhd. Later, he moved to SIRIM Berhad as a researcher before eventually joining MIMOS Berhad in May 2006 as a senior researcher. He is the author of 11 articles and 7 inventions. His research interests include optoelectronics design, optical sensors, phase fluorometry, spectroscopy, quantum randomness, and quantum cryptography.

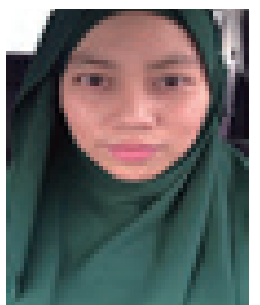

Siti Zuulaika Rejal obtained a bachelor degree in Chemical Engineering from Universiti Malaysia Pahang in 2011 and Master of Science (Fuel Cell Technology) degree in 2015 from Universiti Kebangsaan Malaysia. She was a researcher at MIMOS Berhad. Her research interests include the development of optical dissolved ammonia sensors for agriculture and the development of a direct formic acid fuel cell. 